Harold A. McAlister and William I. Harkopf (eds.)

\title{
Methods of HIPPARCOS Double Star Parameter Estimation for Relative and Absolute Astrometry within the FAST Consortium
}

\author{
H.-H. BERNSTEIN \& R. HERING \\ Astronomisches Rechen-Institut, Mönchhofstraße 12 - 14, D-6900 Heidel- \\ berg 1 , Germany \\ M. BADIALI, D. CARDINI, \& A. EMANUELE \\ Istituto di Astrofisica Spaziale CNR, Via Enrico Fermi 21, I-00044 Frascati \\ (Roma), Italy
}

\begin{abstract}
This paper outlines the details of astrometric parameter determination of double stars from HIPPARCOS observations as prepared in the FAST consortium. Methods of parameter estimation are developed for relative astrometry and absolute astrometry. The capability of the procedures will be demonstrated using simulated observations, in describing the particular steps of a Monte Carlo simulation which have been executed at the Istituto di Astrofisica Spaziale (IAS) and at the Astronomizches Rechen-Institut (ARI).
\end{abstract}

\section{INTRODUCTION}

The aim of ESA's astrometry satellite HIPPARCOS is the determination of positions, proper motions and trigonometric parallaxes of about 120,000 stars. About $40 \%$ of all stars are double or multiple systems. Double stars with separations $\varrho$ in the range of $0^{\prime \prime} .2$ and $5^{\prime \prime}$ and magnitude differences $\Delta m$ less than $3^{m} \cdot 5$ are subject of our analysis (see Dommanget 1985).

Measuring a large basic angle between two different stars by means of two combined telescopes and using a grid in the joint focal plain during the satellite's scanning of the sky yields the star intensity versus a field coordinate per slit. Depending on the geometry of scanning, one observes two or more maxima of the intensity registration as shown in Figure 1.

For each star and each scan a harmonic analysis of the intensity registration (light curve) is performed. In general, the results of this analysis for scans are summarized in the great circle reduction and the spherical coordinates $\psi$ and $\zeta$ (named abscissa and ordinate) are obtained on a corresponding reference great circle (RGC). After fixing all these RGC's on the sky (sphere solution), the determination of the astrometric parameters of each star is the last task in the HIPPARCOS reduction chain. More details of this data reduction are given by Kovalevsky (1980) and Bernacca (1985). A variation of the ratio of the amplitudes of the first and second harmonic of the light curve as well as their phase differences lead to the special treatment of double and multiple stars in the FAST consortium. Here the results of the harmonic analysis of the light curve are supplied to the data condensation and the determination of the parameters of the relative astrometry, whereas the last task of this chain is focused on the absolute astrometry. 

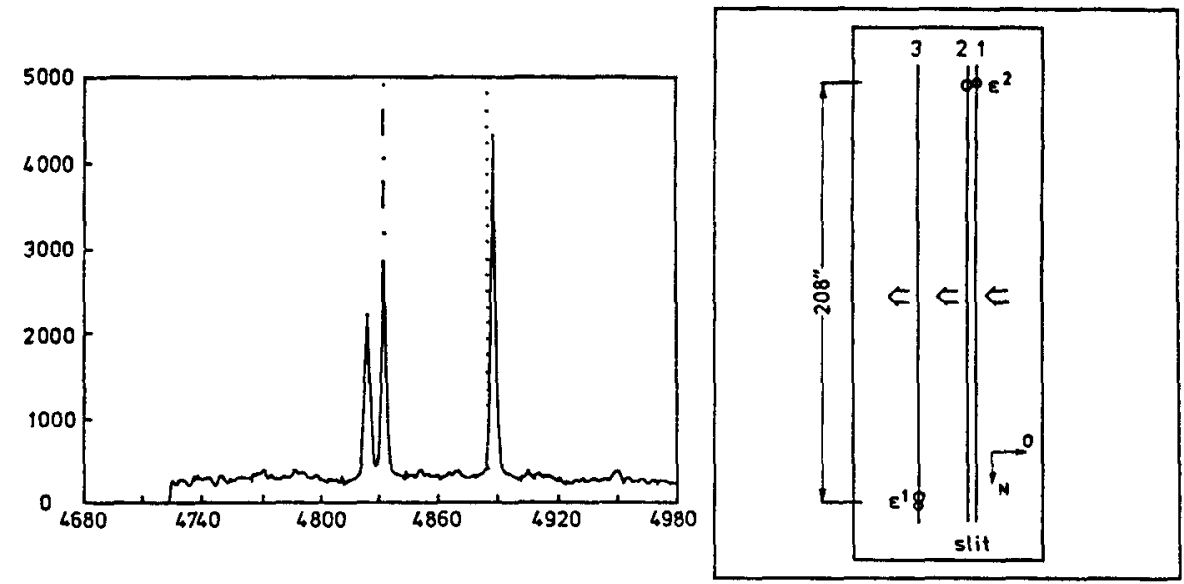

FIGURE 1. The intensity registration of $\varepsilon$ Lyrae versus a field coordinate and the corresponding geometry of scanning (by courtesy of Bastian 1990).

\section{THE ALGORITHM FOR DOUBLE STAR ANALYSIS.}

Here we present only the main steps of the algorithm which, starting from the HIPPARCOS measured signal, gives the relative astrometry parameters for each observed double star system, as well as it's abscissae corrections on each RGC.

\subsection{Data Compression:}

When a double star crosses the field of view, the resulting signal is given by the superposition of the intensities of two single-star signals:

$$
S(t)=S_{1}(t)+S_{2}(t)
$$

with $S_{i}(t)=I_{i}+I_{i} M_{i} \cos \left(\omega t+\phi_{i}\right)+I_{i} N_{i} \cos \left(2 \omega t+\eta_{i}\right)$ where $i=1,2$ is the star index (the quantities of the compound signal have no index), and where $I$ is the total intensity, $M$ and $N$ are the modulation coefficients of the $1^{\text {st }}$ and $2^{\text {nd }}$ harmonics respectively, $\phi$ and $\eta$ are the phases of the two harmonics.

Assuming for each component $\eta_{i}=2 \phi_{i}$, the equation (1) leads to the basic relationships:

$$
\begin{aligned}
I M \cos \phi & =I_{1} M_{1} \cos \phi_{1}+I_{2} M_{2} \cos \phi_{2}, \\
I M \sin \phi & =I_{1} M_{1} \sin \phi_{1}+I_{2} M_{2} \sin \phi_{2}, \\
I N \cos 2 \beta & =I_{1} N_{1} \cos 2 \phi_{1}+I_{2} N_{2} \cos 2 \phi_{2}, \\
I N \sin 2 \beta & =I_{1} N_{1} \sin 2 \phi_{1}+I_{2} N_{2} \sin 2 \phi_{2},
\end{aligned}
$$

with $I=I_{1}+I_{2}, \beta=\phi / 2$.

On the left hand side of Equations (2) there are the observed quantities $(I M, I N, \phi, \beta)$. So, for each frame a set of four values is available. There 
are nine or ten frames per transit across the grid, where a frame is a period of constant satellite attitude. The four phases on the right hand side of eqs. (2) change from one frame to the following as the star moves across the grid. However, the differences $\phi_{2}-\phi_{1}, \phi-\phi_{1}$ and $2 \beta-\phi_{1}$ depend only on the structure of the double star and do not change over the transit. By using this fact and by referring the eqs. (2) to the phases of the $1^{\text {st }}$ harmonic $\phi$, it is possible to write the equations as follows:

$$
\begin{aligned}
& A=I M=I_{1} M_{1} \cos \varepsilon_{1}+I_{2} M_{2} \cos \varepsilon_{2}, \\
& B=0=I_{1} M_{1} \sin \varepsilon_{1}+I_{2} M_{2} \sin \varepsilon_{2}, \\
& C=I N \cos 2(\beta-\phi)=I_{1} N_{1} \cos 2 \varepsilon_{1}+I_{2} N_{2} \cos \varepsilon_{2}, \\
& D=I N \sin 2(\beta-\phi)=I_{1} N_{1} \sin 2 \varepsilon_{1}+I_{2} N_{2} \sin \varepsilon_{2},
\end{aligned}
$$

where $\varepsilon_{1}=\phi_{1}-\phi, \varepsilon_{2}=\phi_{2}-\phi$.

In this form, the equations are invariant over one transit and a compression of data is possible by combining the ten frames into a unique set of parameters, using a minimum $\chi^{2}$ method (Mignard et al. 1989).

\subsection{Solution on the Grid:}

The problem is to invert eqs. (3) from $A, B, C, D$ to the parameters of each component. There are six unknowns and four equations. We can eliminate two unknowns by using the fact that the ratios $(I M)_{i} /(I N)_{i}$ are practically constant. There remains a system of four non-linear equations and four unknowns, which is solved by two iterative methods in sequence: the Newton-Raphson method and the method of steepest descent. This solution is given for each transit separately (Mignard et al. 1986).

Since each transit supplies four new equations, but only two new unknowns $\varepsilon_{1}$ and $\varepsilon_{2}$, after $n$ transits we get $4 n$ equations and $4+2 n$ unknowns. It is possible to fit the whole system of $4 n$ observations to the $4+2 n$ unknowns by a least-squares minimization, using the solutions found at each transit as a starting point to the fitting process. As a result we obtain optimized solutions.

The phase differences on the grid $\varepsilon_{1}$ and $\varepsilon_{2}$, corresponding to some abscissae corrections $\Delta \psi$ on the sky, are necessary to compute both the relative and absolute astrometry parameters.

\subsection{Reconstruction on the Sky:}

Taking into account a double star model on the sky, with a "true" separation $\varrho$ and a "true" position angle $\vartheta$, we have on the tangent plane

$$
x=\varrho \sin \vartheta, \quad y=\varrho \cos \vartheta .
$$

The projected separation on a scan circle is $\Delta S=x \sin \gamma+y \cos \gamma$, where $\gamma$ is the scan angle. Speaking in terms of phase differences $\Delta \phi=\varepsilon_{2}-\varepsilon_{1}$ the projected separation is given by

$$
\Delta \phi / 2 \pi=\Delta S+k
$$

where $k$ is any integer number, accounting for the grid step ambiguity. 
The last equation is equivalent to the following two relationships: $\cos (\Delta \phi)=$ $\cos (2 \pi \Delta S), \sin (\Delta \phi)=\sin (2 \pi \Delta S)$ that with (4) lead to

$$
\begin{aligned}
& \cos (\Delta \phi)=\cos (a x+b y), \\
& \sin (\Delta \phi)=\sin (a x+b y),
\end{aligned}
$$

where $a=2 \pi \sin \gamma$ and $b=2 \pi \cos \gamma$.

When $n$ observations with different values of $\gamma$ are available, one searches the values $x$ and $y$ that are best fitted to the observations by using the deepest hole method (Mignard et al. 1989). The computation takes into account different possible models of relative motions of the two components.

\subsection{Relative Astrometry Optimization:}

This step is also a fitting process between a double star model and the observed signal. It works also for multiple star systems as an independent algorithm. In the case of double stars, the solution achieved by the above described process can be used as a good starting point. In the new fitting process the phases are related to the phase $\phi_{1}$ of the primary star, as follows:

$$
\begin{aligned}
V & =I M \cos \left(\phi-\phi_{1}\right)=I M_{1}+I_{2} M_{2} \cos \left(\phi_{2}-\phi_{1}\right) \\
W & =I M \sin \left(\phi-\phi_{1}\right)=0+I_{2} M_{2} \sin \left(\phi_{2}-\phi_{1}\right) \\
Q & =I N \cos 2\left(\beta-\phi_{1}\right)=I N_{1}+I_{2} N_{2} \cos 2\left(\phi_{2}-\phi_{1}\right) \\
R & =I N \sin 2\left(\beta-\phi_{1}\right)=0+I_{2} N_{2} \sin 2\left(\phi_{2}-\phi_{1}\right) .
\end{aligned}
$$

Unlike eqs. (3), here the left-hand side contains the unknowns $\phi_{1}$ and is not fully determined by the observed signal. But, taking into account the relationship with the quantities $A, C$ and $D$ from (3) $I M \equiv A=\sqrt{V^{2}+W^{2}}$; $I N=\sqrt{C^{2}+D^{2}}=\sqrt{R^{2}+Q^{2}}$, and the expression for the phase difference

$$
\beta-\phi_{1}=\frac{1}{2} \arctan \left\{\frac{\left[R\left(V^{2}-W^{2}\right)-2 Q V W\right]}{\left[Q\left(V^{2}-W^{2}\right)-2 R V W\right]}\right\}
$$

it is possible to search the best fit of the sky model with the observed data by an iterative process. The result is an optimized solution of the relative astrometry parameters with respect to the solution obtained at the last step (Mignard et al. 1989). The relative astrometry parameters, as well as the phase differences, are stored in the DSR file. DSR contains the separation and position angle of the secondary component with respect to the primary, respectively the rectangular coordinates and some possible time derivatives or orbital elements are given. All observed quantities are given with their covariance matrix.

\section{PARAMETER DETERMINATION OF THE ABSOLUTE ASTROMETRY.}

Starting from the measurements, the first step of the absolute astrometry task is to look for a solution of the relative and absolute grid step ambiguity problem. Due to uncertainties of the astrometric parameters of the given input catalogue, all abscissae could be polluted by missing grid steps. Owing to the methods of 
reconstruction on the sky, the relative coordinates show no grid step ambiguities. This fact is used to solve the relative grid step ambiguity problem in the abscissae differences $(\Delta \psi)$ by a comparison with the separation projected on the RGC. This transformation will be done by using the scan angle of the RGC and the position angle of the star. At first we correct the abscissae of the secondary component. After that we use the grid step procedure of Bastian (1985) for the solution of the absolute grid step problem and we correct the abscissae of the primary component. If we supply these corrections to the corresponding abscissae of the secondary the ambiguity problem is solved. As uncertainties in the star position are responsible for the absolute grid step problem, the above mentioned method of Bastian (1985) deals with the comparison of the observed abscissae with a set of synthesized sine curves. In doing so the best fitted set of grid step corrected abscissae solves this problem by a trial and error iteration.

Taking advantage of the symmetry of the satellite's scanning law to the ecliptic, the unknowns are estimated in the ecliptical coordinate system. In general one determines the position, the proper motion, the parallax and, if casually, the acceleration of each component. The parametrization of the absolute astrometry depends on different double star models. According to these different models, introduced by the relative astrometry task, a model one double star is determined by the individual positions of the components, the common proper motion, the common parallax without any acceleration. A model two double star differs from model one by the introducing individual proper motions for the components and for a model three double star we estimate two accelerations additionally. In the case of orbital double stars we estimate the classical five parameters for the gravity centre and the masses of each component.

Due to the statistical nature of the data, the estimation procedure should be an adjustment process with correlated observations. In principle, both sets of abscissae carry information about the absolute astrometry. Especially when $\Delta m \rightarrow 0$ no component should be preferred but for large $\Delta m$ the primary predominates. So the adjustment procedure should be a symmetric approach for $\Delta m \rightarrow 0$ and becomes more one-sided when $\Delta m$ increases. As the development of $\Delta \psi$ does not need actually the knowledge of the parameters of the relative astrometry, the third Kepler law is not considered when the masses should be determined dynamically. So, for orbital double stars, we add the third Kepler law as a constraint to our adjustment procedure. HIPPARCOS measurements results in an observational catalogue of stars. So no external measurements should enter the estimation procedure. But for some critical points as the grid step problem, it could be very helpful to regard external direct measured unknowns during our adjustment to overcome such a problem and to re-adjust the HIPPARCOS data afterwards without these external quantities. In particular we have

$\mathrm{m}$ equations of improvements

for all abscissae:

n equations of improvements

for direct measured unknowns: $\quad \mathbf{v}_{x}=\mathbf{R} \mathbf{x}-\mathbf{l}_{x}$ with $\mathbf{Q}_{x}$ and

c constraints between the unknowns: $\mathbf{0}=\mathbf{C x}+\mathbf{w}$.

Here $\mathbf{l}$ is the vector of observed minus computed abscissae, $\mathbf{A}$ is the matrix of coefficients containing all partial derivatives of $\psi$ with respect to the 
unknowns, $\mathbf{v}$ is the vector of improvements and $\mathbf{Q}_{l}$ denotes the corresponding covariance matrix. Furthermore, $x$ is the vector of solution, $l_{x}$ is the vector of direct observed minus a priori computed unknowns, $\mathbf{R}$ is the coefficient matrix connecting $l_{x}$ and $x, v_{x}$ contains the improvements of these measurements and $\mathbf{Q}_{\boldsymbol{x}}$ is the covariance matrix of $\mathbf{l}_{\boldsymbol{x}}$. At last, for the linearized constraint $\mathbf{w}$ is the vector of inconsistancies and $\mathbf{C}$ contains the partial derivatives of $\mathbf{w}$ with respect to the unknowns in question. Following Lagrange we have the condition:

$$
\mathbf{v}^{T} \mathbf{Q}_{1}^{-1} \mathbf{v}+\mathbf{v}_{z}^{T} Q_{z}^{-1} \mathbf{v}_{z}+2 k_{1}^{T}(A x-1-v)+2 k_{2}^{T}\left(R x-l_{z}-v_{z}\right)-2 k^{T}(C x+w)=\Omega=M i n,
$$

see Wolf (1975). $\mathbf{k}, \mathbf{k}_{1}$ and $\mathbf{k}_{2}$ are the Lagrange factors. Applying some algebra we get the normal equations which solves our estimation problem:

$$
\left(\begin{array}{l}
\mathbf{x} \\
\mathbf{k}
\end{array}\right)=\left(\begin{array}{cc}
\mathbf{A}^{T} \mathbf{Q}_{l}^{-1} \mathbf{A}+\mathbf{R}^{T} \mathbf{Q}_{x}^{-1} \mathbf{R} & -\mathbf{C}^{T} \\
-\mathbf{C} & 0
\end{array}\right)^{+} \cdot\left(\begin{array}{c}
\mathbf{A}^{T} \mathbf{Q}_{1}^{-1} \mathbf{l}+\mathbf{R}^{T} \mathbf{Q}_{x}^{-1} \mathbf{l}_{x} \\
\mathbf{w}
\end{array}\right)
$$

In general the normal equation matrix is positive definite but especially the acceleration terms could be poor measurable for a model three double star. In order to handle the current definiteness we use the Moore Penrose inverse in the normal equations, which minimizes the trace of the normal equation matrix, in the case of non-positive definiteness, otherwise we get the same result as using Cholesky or Gauss decomposition. The Moore Penrose inverse is calculated from the spectral representation of the matrix and the eigenvalues are computed using the QL method which shows good numerical stability.

An error budget is given by the inverse normal equation matrix and the rms of unit weight, estimated from $\mathbf{v}, \mathbf{v}_{x}$ with $\mathbf{Q}_{l}$ and $\mathbf{Q}_{x}$. Escpecially the rms of the unknowns $\mathbf{x}$ and the corresponding correlation matrix are given. For small degrees of freedom, the error propagation is considered to give more reliable rms of the adjustment results.

For comparisons with external catalogues we change the basic coordinate system to the equatorial system during the adjustment procedure and the classical quantities $\varrho$ and $\Theta$ are given with their error budget.

The adjustment is embedded in an iteration loop of up to 30 passages, which enables us to consider possible nonlinearities or to handle external information for undetected grid steps. For every passage a $T$-test checks whether a significant difference is detected or not. In each passage a test of discordant measurements is performed using the procedure of Förstner (1986). A $\chi^{2}$ test applied to the residuals of each double star system points out whether a model deviation is detectable or not.

\section{THE DATA SIMULATION CHAIN.}

In order to test the capability of these procedures, a Monte Carlo simulation was performed including both tasks at IAS and ARI. The basic data sets are taken from Froeschle (1986) and consist in following files:

1. The location of all RGC's, part of a file called mission control general.

2. The origins of these RGC's obtained in sphere solution.

3. A file containing the $a$ priori known astrometric parameters of every star. 
4. The file supplying with abscissae $\psi$ and the ordinate $\zeta$ on each RGC on which the star in question was observed. In the case of double or multiple stars, it contains $\psi$ and $\zeta$ of a reference point, which is defined in the harmonic analysis of the light curve.

5. In order to determine the astrometric parameters of the star's components, one needs the abscissae differences $\Delta \psi$ of these components with respect to the reference point. The $\Delta \psi$ on each $\mathrm{RGC}$ are obtained from the phase differences contained in the DSR file.

The following steps outline the double star simulation:

1. Generation of double star configurations in the true sky. They are characterized by the position, proper motion and parallax of both components.

2. Simulation of the phases as an input for the harmonic analysis.

3. Generation of the coefficients $A, B, C$ and $D$ using the relative astrometric parameters deduced from the quantities given in (1), perhaps some noise could be added.

4. Derivation of the offsets of the components on instantaneous scan circles.

5. Projection of these offsets on the RGC's.

6. Derivation of the double star model from the coefficients A,B,C and D.

7. Construction of the result file (DSR). Essentially, it is supplied by the quantities created in (4) and (5).

8. Reconstruction of astrometric parameters and comparison with the true sky.

\section{RESULTS OF THE RELATIVE ASTROMETRY.}

The set of simulated stars have been processed by the algorithms of automatic analysis leading to relative astrometry results. The evaluation of the results has been performed by comparing the input simulated astrometric parameters with the ones obtained from the analysis, as well as by evaluating some internal indexes of goodness of the employed procedures. Namely, these indexes are:

1. a $\chi^{2}$ estimating the goodness of the fitting procedure which reconstructs the position of the components on the sky;

2. a global index of goodness which is given by the weighted sum of the mean square differences between the observed data and the computed ones.

A correct reconstruction of the double star in the sky has been obtained for 94 systems. In the remaining 6 cases, generally characterized by high magnitudes of both components, the results were far from the input data and the indexes of goodness showed bad values.

Considering the 94 successful results, one can say that the achieved precision is satisfactory. The average errors in separation and in position angle are respectively 2.6 mas and 0.37 . The precision, both in terms of residuals and of internal indexes of goodness, decreases with the growth of the magnitudes of the components and with the growth of the magnitude difference between the components. 


$$
\Delta \varrho(m a s) \quad \Delta \vartheta(\text { degrees })
$$

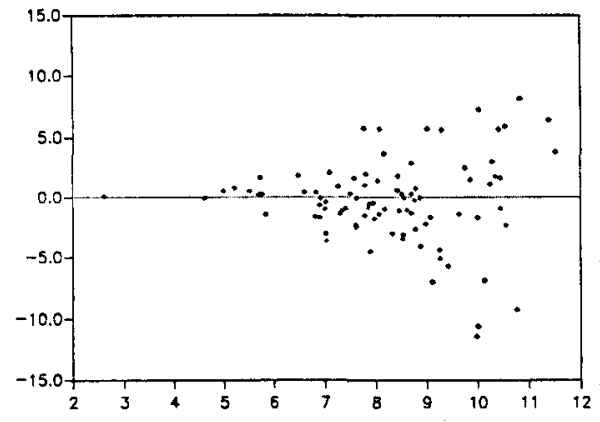

magnitude of the primary

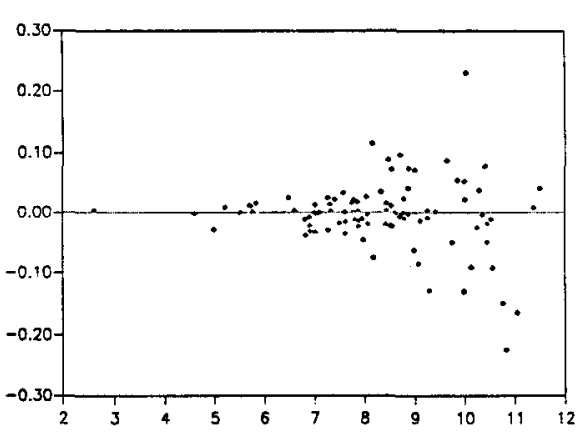

magnitude of the primary

FIGURE 2. Deviations of relative astrometry parameters versus the magnitude of the primary.

In the figures for each star the differences are plotted between quantities (separation on the left and position angles on the right) used as an input in the simulation process and the corresponding results of the analysis. The growth of the error is well visible with the growth of the magnitude. Within the considered ranges of values of other parameters, slighter dependencies were found from the magnitude difference between the components and from the separation.

\section{RESULTS OF THE ABSOLUTE ASTROMETRY.}

The output of the absolute astrometry comprises the positions of the individual double star components, the proper motion of these components or of the star system, the parallax of the system and, if measureable, the acceleration terms of both components. In the case of orbital double stars the classical 5 parameters are given for the gravity centre and for an optical double we give these 5 parameters for both different stars. Additionally we derive the polar coordinates of the secondary with respect to the primary. In accordance to the single star output, the detailed output is expressed in ecliptic coordinates. All quantities are provided with their rms (a priori definition and a posteriori estimation of the error of unit weight) and the corresponding correlation matrix. Details of numerical stability and statistical test results are also part of the output.

In general, the capability of all procedures can be shown by the comparison of the obtained results with the true sky which was the starting point of our simulation loop. 

$\Delta \lambda_{P / S}($ mas $)[\bullet /+]$
$\Delta \beta_{P / S}($ mas $)[\bullet /+]$

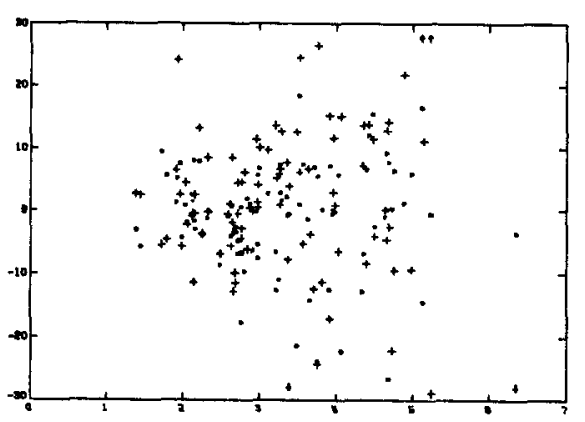

$\varrho(")$

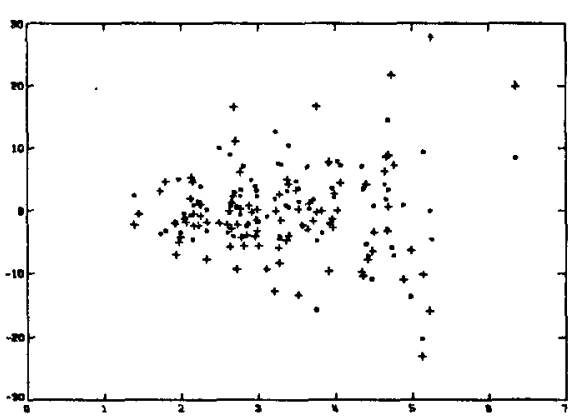

$\varrho(")$

FIGURE 3. Deviations of astrometric parameters versus separation.

The table on the right side contains the mean deviations of the astrometric parameters of double stars with fixed components, obtained from the adjustment procedure, to the corresponding values of the true sky with their rms. Considering the double stars' error budget and using the rms ratio of the measurements, the adjustment results agree with the true sky within the well known limits of the FAST single star treatment (see Lenhardt et al. 1991), so the capability of the shown method is confirmed.

\begin{tabular}{c|c|}
\hline \multicolumn{2}{|c|}{$\begin{array}{c}\text { True Sky - Adjustment Results } \\
\text { (based on 90 star systems) }\end{array}$} \\
\hline Par. & Mean Deviation \\
\hline$\lambda_{P}$ & $-2.47 \pm 0.14 \mathrm{mas}$ \\
$\beta_{P}$ & $+1.71 \pm 0.09 \mathrm{mas}$ \\
$\mu \lambda$ & $+2.11 \pm 0.12 \mathrm{mas} / \mathrm{y}$ \\
$\mu \beta$ & $-7.56 \pm 0.12 \mathrm{mas} / \mathrm{y}$ \\
$\pi$ & $+5.43 \pm 0.11 \mathrm{mas}$ \\
$\lambda_{S}$ & $-0.77 \pm 0.25 \mathrm{mas}$ \\
$\beta_{S}$ & $-0.92 \pm 0.16 \mathrm{mas}$ \\
\hline
\end{tabular}

Figures 3 and 4 show the deviations $\Delta \lambda$ and $\Delta \beta$ versus $\varrho$ and $\Delta m$ for both components $([\bullet]$ : primary and [+]: secondary component). The growth of deviations in both coordinates per component is similar to the relative astrometry result but the values of the particular deviations seem to be larger by a factor of 2 than those in $\Delta \varrho$. Apparently the scatter is larger for quantities of the secondary component than those of the primary. Additionally to the effect of lower intensities of the secondaries on these results a scatter is introduced in the data simulation chain (RGC reduction), so this behavior is not unexpected. 


$$
\Delta \lambda_{P / S}(m a s)[\bullet /+]
$$

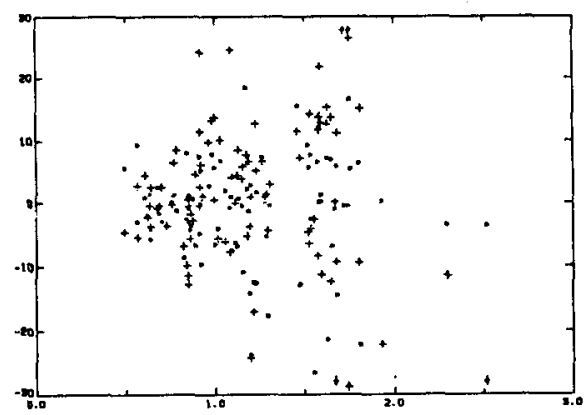

$\Delta m(\mathrm{mag})$

$$
\Delta \beta_{P / s}(\operatorname{mas})[\bullet /+]
$$

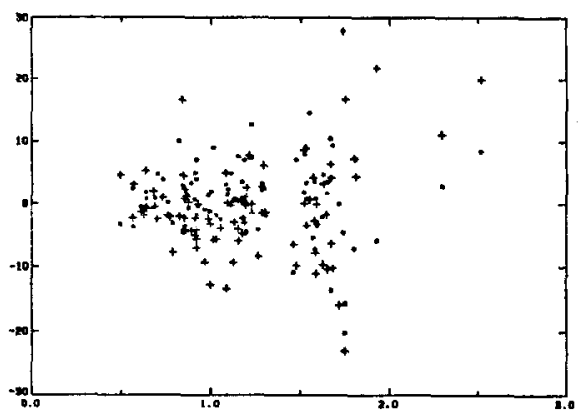

$\Delta m(\mathrm{mag})$

FIGURE 4. Deviations of astrometric parameters versus magnitude difference.

\section{REFERENCES}

Bastian, U. 1985, "Treatment of the Grid Step Ambiguity Problem within Task 6000", in Processing of Scientific Data from the ESA Astrometry Satellite HIPPARCOS, Proceedings of the Second Thinkshop of the FAST Consortium held at Marseille, 21 to 25 Jan. 1985, ed. J.Kovalevsky, (Grasse: CERGA)

Bernacca, P.L. 1985, Project HIPPARCOS, Ap\&SS, 110, 21

Dommanget, J. 1985, HIPPARCOS Astrometric Binaries, Ap\&SS, 110, 47

Förstner, W. 1986, "Statistical Concepts for Quality Control", in International Archives of Photogrammetry and Remote Sensing, (Finland: Rovaniemi), 26, part 3/4

Froeschle M. 1986, Simulation des Donnes pour la Tache "Astrometric Parameter Determination", FAST Technical Note No. 13, CERGA

Kovalevsky, J. 1980, The Hipparcos Space Astrometry Mission, Celestial Mech, 22

Lenhardt, H., Hering, R., \& Walter, H.G. 1991, Procedures for Validating HIPPARCOS Astrometric Parameters, Manuscripta Geodaetica, 16, 254

Mignard, F., Froeschle, M., \& Falin, J.L. 1986, Photometry with IDT II. Double Stars, Proceedings of the Third Thinkshop of the FAST Consortium held at Bari, 3 to 6 Nov. 1986, ed. P.L. Bernacca and J.Kovalevsky.

Mignard, F. et al. 1989, Double Star Reductions in FAST, ESA Sp-1111, Vol. III, p. 243

Wolf, H. 1975, Ausgleichungsrechnung Formeln zur praktischen Anwendung, Dümmler Verlag Bonn 